\title{
Review Article \\ Cavity-Enhanced Spectroscopy in Condensed Phases: Recent Literature and Remaining Challenges
}

\author{
Jonathan E. Thompson \\ Department of Chemistry \& Biochemistry, Texas Tech University, Lubbock, TX 79409-1061, USA \\ Correspondence should be addressed to Jonathan E. Thompson; jon.thompson@ttu.edu
}

Received 14 September 2016; Accepted 18 December 2016; Published 29 March 2017

Academic Editor: Jau-Wern Chiou

Copyright (c) 2017 Jonathan E. Thompson. This is an open access article distributed under the Creative Commons Attribution License, which permits unrestricted use, distribution, and reproduction in any medium, provided the original work is properly cited.

\begin{abstract}
Recent developments in the field of cavity-enhanced spectroscopy (CES) on liquid samples are presented. The various experimental approaches to achieving the measurements are summarized, and the limitations of each approach are discussed. I conclude CES is most promising for microscale light absorption measurements on submicroliter volumes of fluid.
\end{abstract}

\section{Introduction}

Analytical chemistry requires sensitive and broadly applicable measurement technologies to continue to push the boundaries of chemical knowledge. While techniques such as fluorescence and amperometry provide highly sensitive measurements, only a select number of analytes are fluorescent or electrochemically active. Absorption spectroscopy is an attractive alternative since a large number of molecules absorbs light in either the visible or ultraviolet region of the spectrum. However, absorption spectroscopy fundamentally must determine a small difference between two largemeasured quantities (e.g., incident irradiance and sample irradiance) which can prove difficult. The simile "absorption spectroscopy is like trying to determine the weight of a ship's captain by measuring the weight of his boat with and without him" has been attributed to the Stanford scholar R. N. Zare as a means to describe absorption spectroscopy. Despite the inefficiencies, absorption spectroscopy remains a staple of the modern laboratory.

In recent years, several research groups have begun the exploration of cavity-enhanced spectroscopy (CES) as a means to improve limits of detection for absorption spectroscopy for liquid samples. Cavity-enhanced techniques take several different forms, but all have in common the use of an optical resonator within which a sample of light is added. The resonator can take the form of a mirrored optical cell, or an optical fiber loop. In all cases, the experimentalist attempts to create very large effective path lengths to improve sensitivity. In this manuscript, I briefly describe the array of CES techniques recently developed for the liquid phase, summarize several experimental challenges, and conclude that CES may have a bright future for absorption measurements on microvolumes of fluids.

\section{The Three Modes of CES}

2.1. Cavity Ring-Down Spectroscopy (CRDS). Ring-down spectroscopy evolved from the need to measure the reflectance of high-reflectivity dielectric mirrors. The mirrors are constructed from many $\lambda / 4$ layers of materials of varying refractive index such that reflections constructively interfere at interfaces. Two such low-loss mirrors aligned with one another (see Figure 1) can form an optical resonator such that a pulsed beam reflects between the mirrors many hundreds or thousands of times. On each pass thru the resonator, a fraction of the beam will be lost due to limitations of the mirror reflectivity and optical loss due to absorption or scattering within the optical cell. In the experiment, a fast detector is used to track the power of the light within the resonator in time. Any particular cavity mode will decay according to first-order exponential decay kinetics (see Figure 1) and the time constant $(\tau)$ for the decay measured through curve fitting routines. The time constant is referred to as the ringdown time, and its value depends upon the mirror reflectivity and optical losses. If mirror reflectivity remains constant 


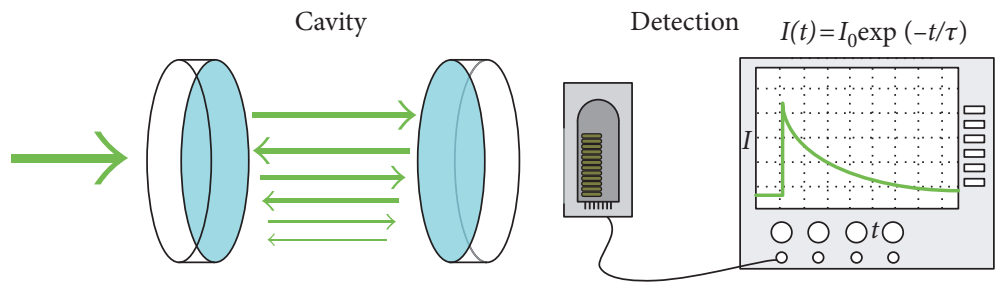

FIGURE 1: Experimental Setup for CRDS. Figure reprinted from [26].

during experiments, change in ring-down time can be linked to increased absorption or scattering within the sample placed between the mirrors. CRDS has long been used to measure gas-phase absorbers [1-3] or aerosols [4-8] since optical losses due to scattering in the gas phase is minimal $[9,10]$ and effective path lengths of kilometers can be achieved. In addition, since the measurement monitors the rate of light lost from the cell, the measurement is independent of spectroscopic source power fluctuations. These effects lead to highly sensitive analysis, with extinction coefficients $<10^{-12} \mathrm{~cm}^{-1}$ being measureable in extreme experimental cases [11].

2.2. Integrated Cavity Output Mode (ICOS). While CRDS measurements use a pulsed light source, a second mode of cavity-enhanced measurements uses an optical resonator with a continuous-wave light source. Intercavity power is monitored and related to sample absorption $(\alpha)$ through

$$
\frac{I_{0}}{I}=1+\frac{\alpha l}{1-R}
$$

where $I$ is the intercavity power during measurements, $I_{0}$ is the power for a spectroscopic blank, $l$ is the cavity length, and $R$ is the mirror reflectivity (1) [12]. This ICOS mode offers the advantage of being able to use spectroscopic sources and detectors that have rise/response times greater than the nanosecond range typically required for CRDS mode while maintaining large path lengths. Another major implication of ICOS is the option of using continuum sources to perform broadband spectroscopy. Just like CRDS mode, many investigators have explored ICOS for gasphase and aerosol measurements [13-15].

2.3. Cavity Attenuated Phase Shift Spectroscopy (CAPS). A third variant of CES is cavity attenuated phase shift spectroscopy. In this approach, the intensity of a $\mathrm{cw}$ source is modulated at a frequency $f$ on a timescale similar to the cavity ring-down time $(\tau)$. Reid et al. [16] indicate the presence of the cavity will induce a measureable phase shift between the input and output signals of

$$
\phi=-\arctan (f \tau)
$$

Determination of the phase shift leads to knowledge of $\tau$ and subsequent determination of sample absorption. Just like CRDS and ICOS modes, cavity attenuated phase shift measurements have typically been applied to gas-phase and aerosol measurements [17-19].

\section{CES Experiment Design}

3.1. Liquid Phase CES Experiments within Linear Resonators. The most obvious experimental apparatus for extending the CES technique into condensed phases is to simply place the liquid or solid phase sample within a linear optical resonator. The liquid sample itself must be contained within a cuvette, a chamber formed between the high-reflectivity mirrors, or a liquid sheet or jet. Figure 2 illustrates four experimental designs that have been pursued for CES in the condensed phase. In design A, a sample-filled cuvette is held at Brewster's angle within an optical cell to minimize reflective losses at the air-glass interface. In design $\mathrm{B}$, the liquid sample is placed directly in contact with the HR mirrors. In design C, a commercial cuvette is placed at normal incidence within the resonator. While this geometry leads to higher reflection at the air-glass interface compared with design A, with careful alignment, the reflected light can return to the mirror and continue circulating in the cell. In design $\mathrm{D}$, sample liquid is sprayed onto a wedge that causes the production of a thin sheet of sample liquid. This thin layer is then probed via CES at the Brewster angle.

Due to simplicity, the most popular experimental design places a cuvette within the resonator. Islam et al. tested three sets of high-reflectivity mirrors and a $2 \mathrm{~mm}$ quartz cuvette while using high-intensity visible LEDs as light sources for ICOS-CES experiments [20]. For samples containing either $\mathrm{Ho}^{3+}$ or organic dyes, the authors found path length enhancements as high as 104-fold (also known as cavity enhancement factor) due to the optical resonator. This led to minimum detectable absorbance in the range of $0.00005-$ $0.0012 \mathrm{~cm}^{-1}$ over the spectral range tested $(420-670 \mathrm{~nm})$. The sensitivity enhancement was limited by the scattering losses per pass that were reported as being approx. 0.01 per pass. In later work, the authors removed the cuvette windows and increased the path length of the cell to $20 \mathrm{~cm}$, which lowered the detection limit of the system to $3 \times 10^{-7} \mathrm{~cm}^{-1}$ [21].

One of the unique features of broadband CES is the ability to rapidly collect absorbance spectra across several hundred nanometers. However, the mirrors used in CES do not exhibit uniform reflectivity across the entire spectral region, and overtones of fundamental $\mathrm{CH}$ and $\mathrm{OH}$ stretching frequencies from solvents will cause differing optical loss as a function of wavelength. The consequence of this is that the effective absorption path length (or cavity enhancement factor) is not identical for all wavelengths. This results in distortion of absorption spectra compared to the case of a uniform path length. Figure 3 shown below from Seetohul et al. [21] illustrates cavity enhancement factor can vary 


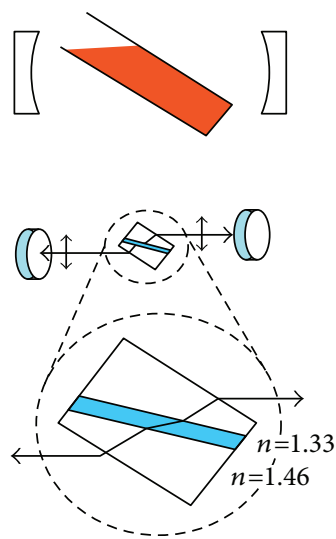

(a)

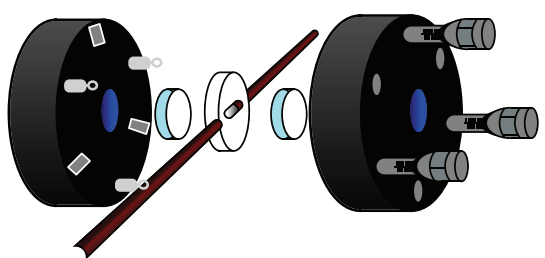

(b)



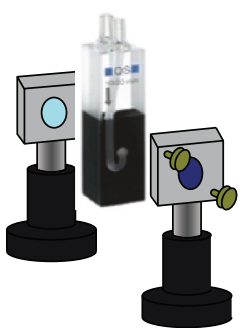

(c)

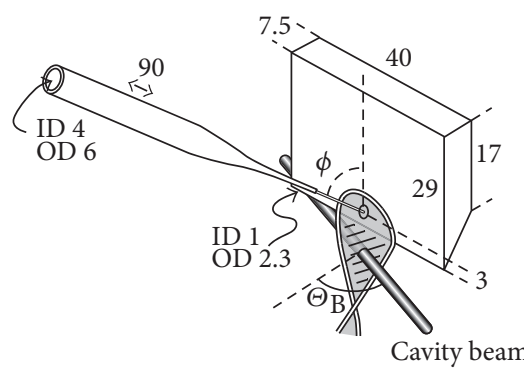

(d)

FIGURE 2: Experimental designs for implementing cavity-enhanced spectroscopy (CES) within mirrored resonators. (a) represents a design in which the cuvette holding the sample is placed at Brewster's angle to minimize reflective losses. (b) Flow thru cuvette in which the mirrors form the windows of the optical cell. The mirrors are placed in direct contact with the solvent. (c) Two-mirror resonator in which the sample is contained within a commercial flow-through cuvette. $(a-c)$ are reproduced with permission from [26]. (d) Liquid sheet jet for condensed phase CES. Liquid is sprayed onto a wedge causing the production of a thin sheet of liquid that is probed at Brewster's angle via CES. Image reprinted with permission from Alexander [47]. Copyright 2006 American Chemical Society.

3-fold as a function of wavelength. The absorption spectrum collected by the CES spectrometer is consequently distorted as illustrated in Figure 3(b). The broad blue trace in Figure 3(b) is the single pass spectrum for a solution of Sudan black, while the additional traces are recorded spectra within differing solvents. Notice that the reported absorption during the CES experiments dip at approx. $500 \mathrm{~nm}, 550 \mathrm{~nm}$, and $625 \mathrm{~nm}$. These absorption features are not accurately depicted in the CES absorption spectrum but are artifacts of the cavity enhancement factor dipping at the same wavelengths (see Figure 3(a)). Consequently, the spectra reported by CES must be mathematically corrected to yield quantitatively accurate reports of absorption.

A further complication of CES exists because the effective path length is also a function of the per-pass optical loss as light cycles in the resonator. Consequently, the measured absorbance at any particular wavelength is a nonlinear function of absorber concentration. This nonlinear behavior is most obvious under experimental conditions in which the optical absorption loss dominates the total per-pass losses. The nonlinear behavior is shown in Figure 4 from
Seetohul et al. [21]. This adds additional difficulty to quantitative analysis using CES.

Despite the limitations, many other investigators have also pursued CES within linear cavities. Kiwanuka et al. [22] used supercontinuum radiation and a $30 \mathrm{~cm}$ long linear optical cavity formed from broad band mirrors with $R>99 \%$ between approx. 400-680 $\mathrm{nm}$ to perform CES on liquid samples placed within a cuvette. The $5.4 \mathrm{~cm}$ long cuvette held $2.7 \mathrm{~mL}$ of fluid and had windows that were carefully adjusted to achieve normal incidence of the circulating light beam. Because these authors used a spectrograph, complete broadband spectra could be collected very rapidly-within $50 \mathrm{~ms}$ exposure time. The authors used the apparatus to collect broadband visible absorption spectra of nanomolar concentrations of fluorescent dyes. The authors also demonstrated the potential of the method for the study of chemical kinetics by monitoring the absorption spectrum during the BelousovZhabotinsky reaction. This reaction exhibits periodic oscillations in solution color as cerium(IV) (yellow) ions are reduced to cerium(III) (colorless), followed by cerium(III) cycling back to cerium(IV) through oxidation by bromate. 




(a)

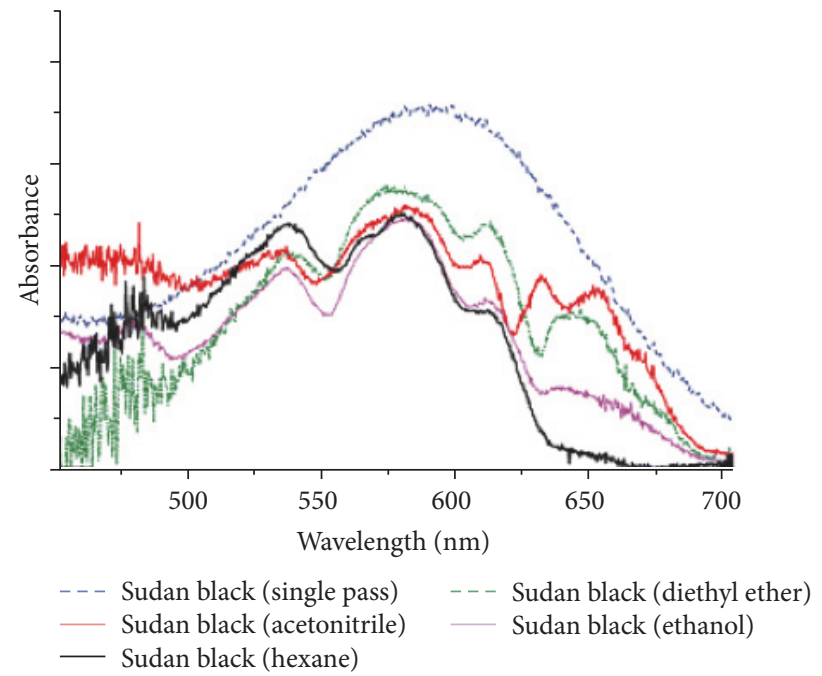

(b)

FIGURE 3: (a) Plot of cavity enhancement factor versus wavelength. (b) Plot of indicated absorbance versus wavelength for Sudan black dye in a variety of solvents. The blue trace is a single-pass absorption spectrum, while the remaining traces are CES experiments. Notice that the CES spectra are distorted at certain wavelengths due to variation in the cavity enhancement factor. Figures reproduced with permission from Seetohul et al. [21].



Figure 4: Nonlinearity of absorbance with concentration during CES. Nonlinearities generally appear under conditions where absorption dominates per-pass optical loss. Figure reproduced with permission from Seetohul et al. [21].

More recent work has focused on applying CES to realworld problems. For instance, Bajuszova et al. have pursued making CES measurements within a resonator that has been engineered to accommodate microtiter plates [23]. This allows coupling of the sensitive CES technique with common bioanalytical diagnostic tests such as ELISA. In Bajuszova et al. the authors use a white-light LED emitting between 400 and $700 \mathrm{~nm}$ to excite a $10 \mathrm{~cm}$ length resonator formed between two HR mirrors with $R>0.99$ across $420-640 \mathrm{~nm}$. An $x, y$ positioner controls a stage that supports and moves standard microtiter plates such that each well can be interrogated serially. The authors report that glass-bottomed microtiter plates introduce optical losses of $20 \%$ and polystyrene plates induce losses of $50-70 \%$. Nonetheless, the authors report a path length enhancement of 30-fold through use of CES and state that the CES approach may begin to approach the sensitivity of fluorescence-based ELISA provided further refinements that can be realized. The significant optical losses induced by the microtiter plates appear to present a significant obstacle to improve sensitivity. In general, optical loss at the air-cuvette interface limits the cavity enhancement factor and sensitivity increase that can be achieved.

Another recent innovation is the work of Arai et al. [24] who used a confocal mirror cavity to image absorption within thin slices of tissue samples and cells. The beam waist of the fundamental Gaussian mode of a mirror resonator can be quite small (micrometer size range), and rastering of the spot across a $2 \mathrm{D}$ surface can allow spatial mapping. In the work of Arai et al., the authors achieved 2D mapping by placing a sample mounted on an $x, y$ piezoelectric stage with $1 \mu \mathrm{m}$ positioning resolution at the center of an optical cavity. The optical source was a fiber-coupled supercontinuum laser providing broadband radiation allowing spectra between 450 and $600 \mathrm{~nm}$ to be collected. The authors report a 15-micron spatial resolution for the mapping of absorption features of the tissue sample and found differences in spectra between cell types using principal component analysis.

3.2. CES in Optical Fiber/Waveguides. Another instrumental variant of CES is to conduct the experiment within a linear section or loop of optical fiber [25, 27-34]. This implementation of CES is attractive because measurements on sub-nL volumes of the sample can be conducted due to the small diameters of optical fibers used. CES within fibers has already been recently reviewed by Waechter et al. [35]. If spectroscopy within a linear fiber is conducted, the ends of the fiber are equipped with either reflective dielectric mirrors [25] or 


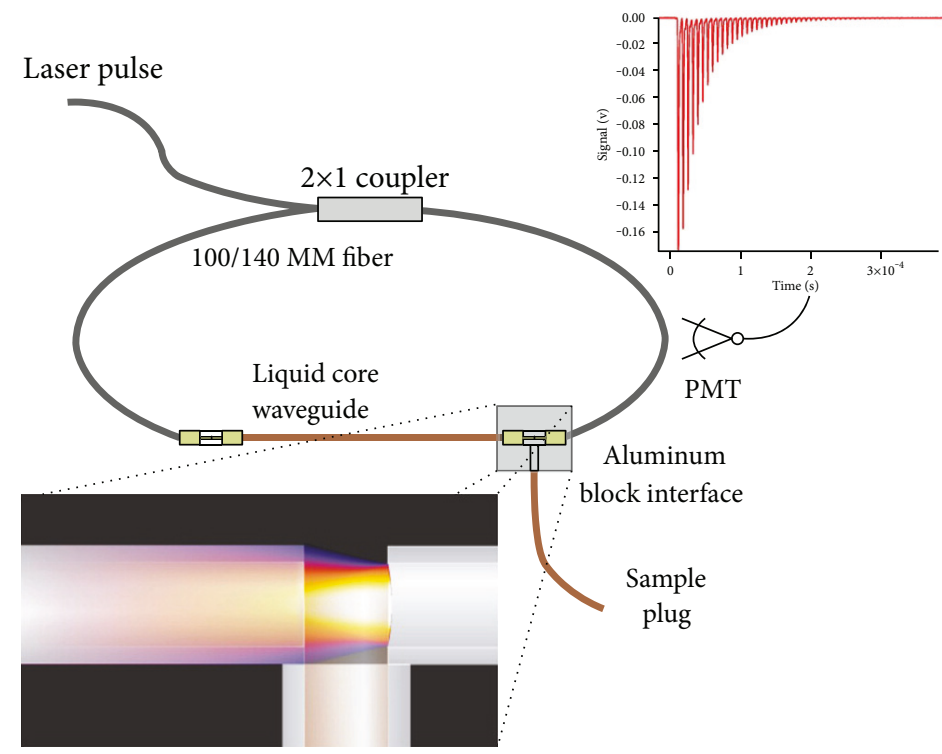

(a)

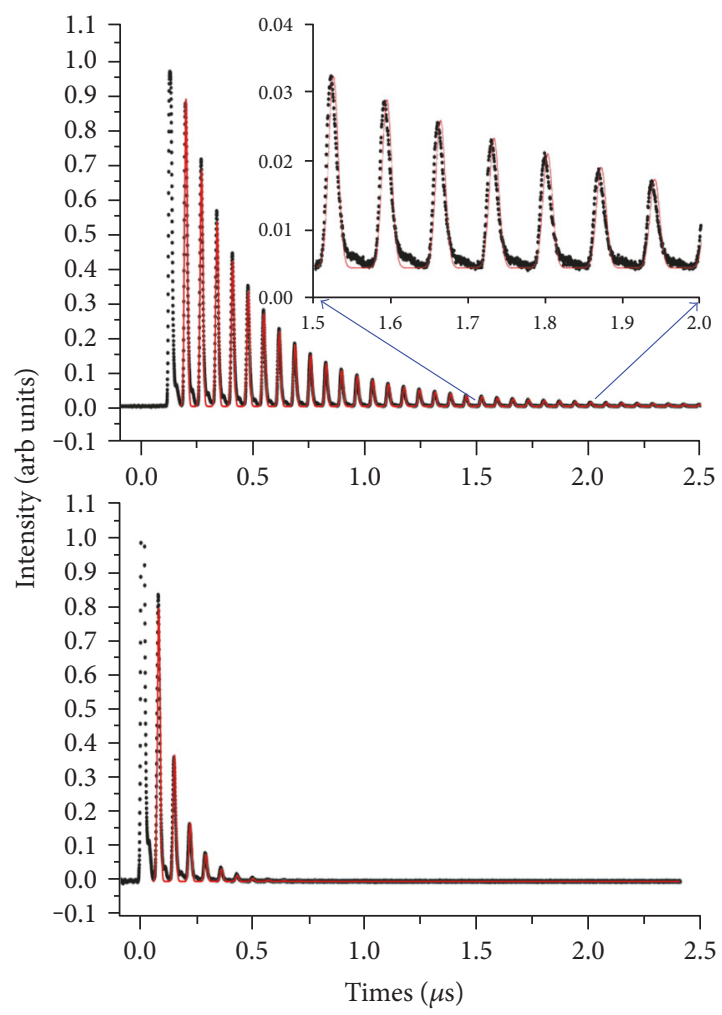

(b)

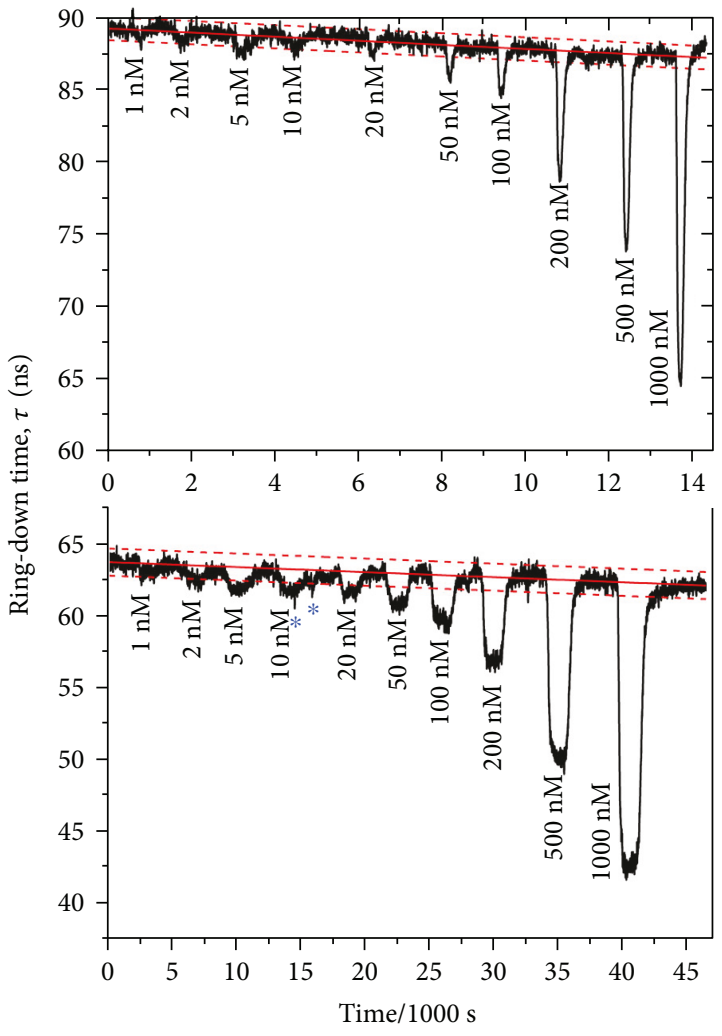

(c)

FIgURE 5: (a) Experimental apparatus for fiber loop CRDS with a liquid core waveguide. (b) Data illustrating ring-down pulses with and without the waveguide being present. Without the waveguide, the round-trip loss was $11.6 \%$. With the waveguide, the round-trip loss increased to $53.9 \%$ (c) Data illustrating recorded ring-down time over the course of an experiment in which $20 \mu \mathrm{L}$ plugs of absorbing dye in the $\mathrm{nM}$ concentration range were sequentially injected into the waveguide. Top trace in (c) is for Allura red and bottom trace is for Congo red dye. Figures have been adapted from Bescherer et al. [31]. Copyright 2013 American Chemical Society.

fiber Bragg gratings to achieve reflection [36]. The Bragg grating approach offers the advantage of high reflection, but only over a narrow wavelength band for which the device was designed (usually wavelengths for Telecom applications). By contrast, a resonator created by looping an optical fiber will circulate broadband radiation and is subject to losses due to optical components (splicers) placed within the loop, the native absorption of the fiber material, and the fiber-bending losses only (also known as macrobending losses). The absorbing sample may be placed within a gap 

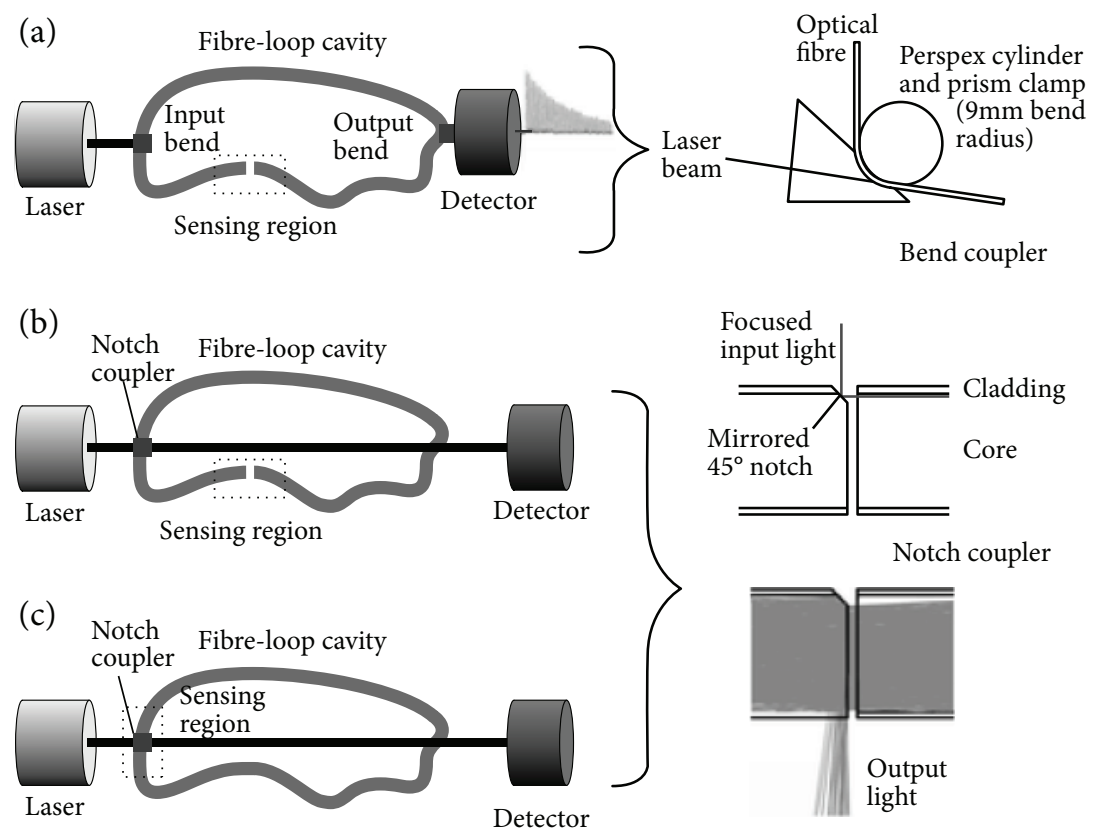

FIGURE 6: Experimental options for introducing light into a fiber loop for CES. (a) Macrobend coupler in which a laser beam is directed at a bend in the fiber and a small fraction of light is coupled through the fiber cladding. ((b) and (c)) Notch couplers reported in Rushworth et al. Figure reprinted with permission from Rushworth et al. [34]. Copyright 2011 American Chemical Society.

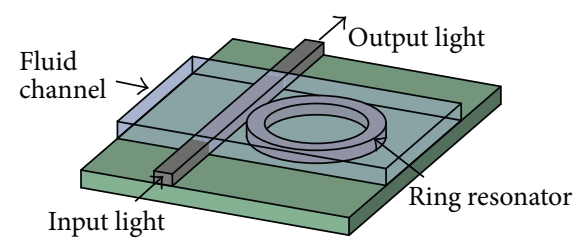

(a)

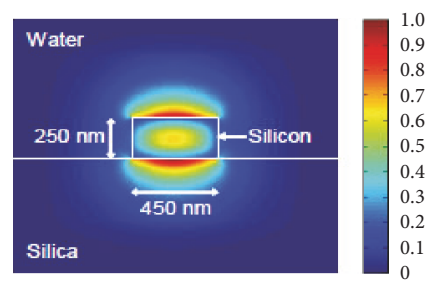

(b)

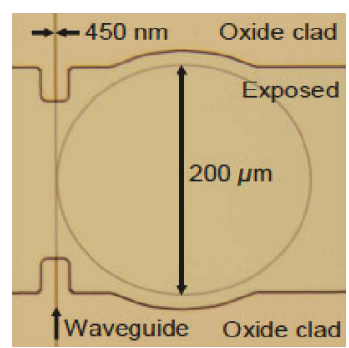

(c)

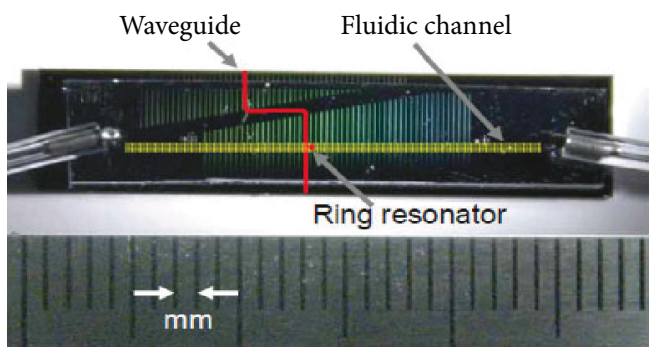

(d)

Figure 7: (a) Schematic of the microring resonator adjacent to the fluid channel placed on top. The color-scaled image in (b) depicts the electric field profile propagating within the waveguide when an aqueous sample is loaded within the device. (c) Optical microscope images of the ring resonator, waveguide, and fluidic chip. Note that the completed device in (d) incorporates 50 closely spaced waveguides and resonators. Figure reproduced from Nitkowski et al. [42].

between fiber ends or alternatively within a waveguide that light circulates through.

Bescherer et al. [31] performed cavity ring-down mode measurements within a 5-10 cm length liquid-core waveguide with excitation of the resonator via the 2 nd harmonic of a Nd:YAG laser. The experimental setup and results are shown in Figure 5. While the analysis was successfully performed on a detection volume $<1 \mu \mathrm{L}$, the optical coupling losses between components caused a $53.9 \%$ optical loss per pass. This high degree of per-pass loss resulted in ring-down times $<100 \mathrm{~ns}$-far shorter than what is typically encountered in gas-phase measurements. Despite the very short ring-down time, the authors were able to achieve a minimum detectable absorbance of $0.0004 \mathrm{~cm}^{-1}$. The loss 
TABLE 1: Review of literature CES methods.

\begin{tabular}{|c|c|c|c|c|c|}
\hline Absorber & Method & $\lambda(\mathrm{nm})$ & Sample volume & Min. det. optical loss $\left(\mathrm{cm}^{-1}\right)$ & Ref. \\
\hline Allura red dye, Congo red & LCW CRDS & 532 & $<1 \mu \mathrm{L}$ & 0.00034 & [31] \\
\hline Malachite green & LJ CRDS & 628 & $150 \mathrm{~mL}$ & 0.016 & [47] \\
\hline Benzene & NICUV-CES & $450-650$ & NA & 0.00002 & [48] \\
\hline Alexa Fluor dye & NICUV-CES & $400-700$ & $2.7 \mathrm{~mL}$ & 0.000009 & {$[22]$} \\
\hline Brilliant blue dye & NICUV-CRDS & 630 & NA & 0.00005 & [20] \\
\hline Rhodamine $6 \mathrm{G}$ & NICUV-CRDS & $500-600$ & $\sim 3 \mathrm{~mL}$ & 0.000065 & [49] \\
\hline Quinalizarin & BRCUV-CRDS & 470 & $10 \mu \mathrm{L}$ & 0.00062 & [50] \\
\hline Benzene & BRCUV-CRDS & $580-660$ & NA & 0.0000002 & [51] \\
\hline Crystal violet & LF-CRDS & 532 & $12 \mu \mathrm{L}$ & 0.00031 & {$[52]$} \\
\hline Bacteriochlorophyll & LF-CRDS & 783 & few $\mathrm{mL}$ & 0.000016 & [53] \\
\hline $\mathrm{Cu}^{2+}$, indigo carmine & LF-CRDS & $620-670$ & $10 \mu \mathrm{L}$ & 0.000001 & {$[54]$} \\
\hline Methylene blue & LF-CRDS & 655 & $60 \mathrm{~mL}$ & 0.00000033 & [55] \\
\hline Sudan black, methylene blue & LF-CRDS & $450-700$ & $100 \mathrm{~mL}$ & 0.0000003 & [21] \\
\hline Tartrazine & FG-CRDS & 405 & $100 \mathrm{~nL}$ & 0.02 & [56] \\
\hline Tartrazine & FG-CRDS & 405 & $6 \mathrm{~nL}$ & 0.11 & [57] \\
\hline Rhodamine & FG-CRDS & 532 & $19 \mathrm{~nL}$ & 0.11 & [34] \\
\hline Permanganate ion & FG-CRDS & 532 & $130 \mathrm{~nL}$ & 0.0024 & [58] \\
\hline Ferrozine complex & LCW & 562 & $300 \mu \mathrm{L}$ & 0.000013 & [59] \\
\hline Bromothymol blue & LCW & 613 & $1 \mu \mathrm{L}$ & 0.02 & {$[60]$} \\
\hline
\end{tabular}

LCW: liquid core waveguide; LJ: liquid jet; CRDS: cavity ring-down spectroscopy; CES: cavity-enhanced spectroscopy; NICUV: normal incidence cuvette; BRCUV: Brewster angle cuvette; LF: liquid-filled cell in which fluid directly contacts fluid; FG: fiber gap; NA: not available.

of light at couplers or junctions between fibers in the loop is a major source of per-pass optical loss for fiber CES experiments. In addition, bending the fiber induces "macrobending" losses in which a fraction of the light no longer maintains the correct incidence to meet the internal reflection criterion [37].

To combat these losses in fibers, Andrews et al. [29] devised a remarkably clever experiment in which a mechanism for light intensity enhancement (gain) is added to the fiber loop on each pass. These authors accomplished nearinfrared CRDS measurements on approx. $1 \mathrm{pL}$ fluid volumes placed within a $19 \mu \mathrm{m}$ gap between fibers. Expanding upon the seminal work of Stewart et al. [38, 39] the work of Andrews et al. [29] used an erbium-doped fiber amplifier within the optical loop to provide gain within the fiber to match the undesirable (nonabsorptive) per-pass losses caused by the apparatus. This innovative approach can help account for the high per-pass losses within the fiber loops; however, it can only be applied to certain wavelengths that the amplifier is designed for. Nonetheless, the authors demonstrated detection of 1-octyne within dodecane solvent at $\lambda=1532 \mathrm{~nm}$ with a minimal detectable absorption of $0.033 \mathrm{~cm}^{-1}$.

One additional difficulty encountered for fiber loop CES experiments is coupling the probe light efficiently into the resonator. This is usually accomplished through fiber couplers, laboratory built interfaces, notches within the fiber's cladding, or even through bends in the fiber itself. As illustrated in Figure 6, Rushworth et al. [34] have demonstrated a novel coupler based upon a reflective surface used to increase coupling efficiency of light into the fiber resonator to, in theory, near $100 \%$ efficiency. In this approach, one end of a fiber is cut and polished to provide a metallized $45^{\circ}$ facet upon which incident light is directed. This light can then be reflected into the core of the other end of the fiber, forming the fiber loop. Reported signal intensities for the notch coupler were at least $40 \times$ greater when compared to the bendcoupling approach. This result is significant as the notch coupler may allow low-power light sources such as lamps or LEDs to be employed for CES within fiber loops as has previously been demonstrated in CRDS mode with mirror resonators [40].

While directly probing molecular absorption by shining a beam through a sample is one experimental route, evanescent wave spectroscopy using fiber resonators is also gaining popularity. Since the evanescent wave extends only a very short distance outside the fiber core, the fiber is usually tapered, side polished, or etched to allow measurement $[25,36,41]$. The evanescent wave approach is attractive since no break in the fiber loop is required for analysis. In turn, this limits nonabsorptive losses and should allow more sensitive analysis.

One demonstration of this approach is the work of Nitkowski et al. who engineered a microring resonator into a microfluidic device [42]. As observed in Figure 7, the ring resonator was optically coupled to a tunable near-infrared laser $(1460-1610 \mathrm{~nm})$ via a waveguide. A fluid sample was passed over the ring resonator through a channel fashioned into polydimethylsiloxane (PDMS). The sample fluid serves as the cladding of the resonator. Absorption of the evanescent wave will lead to increased losses and will be sensed. 
The authors have reported an absorption spectrum of $\mathrm{N}$ methylamine near $1500 \mathrm{~nm}$ using the microring device. This technology is promising due because the CES is directly coupled to microfluidic devices that are becoming increasingly popular in bioanalytical chemistry for chemical and cellular analysis [43-45]. In addition, this type of integrated device has significant promise for rapidly screening purity and yield of reaction products from the laboratory or classroom [46]. In Nitkowski et al., the absorption spectrum can be made on a sample volume of roughly $2 \mathrm{~nL}$-approximately a one millionfold decrease compared to conventional spectrophotometers.

\section{Summary and Prospects}

In recent years, cavity-enhanced spectroscopies have been demonstrated in condensed phases. Initial work has often mimicked the experimental apparatus for gas-phase measurements by using mirrored, linear resonators. This approach has allowed investigators to probe optical absorptions in the $10^{-5} \mathrm{~cm}^{-1}$ range. However, optical losses at the air-cuvette interface and scattering from liquid samples limit the cavity enhancement factors achievable. In addition, modern commercial spectrophotometers also report detection limits in the $10^{-5} \mathrm{~cm}^{-1}$ range with a simpler and much more userfriendly apparatus. Therefore, it appears as if the main analytical advantage of CES for future applications are those in which the probed sample volume can be reduced dramatically. Commercial spectrophotometers typically make measurements on samples in the $1-3 \mathrm{~mL}$ range. As reported in Table 1, several investigators have been able to either miniaturize a 2-mirror resonator or use fiber CES to achieve sample volumes of microliters or less. When the sample volume is reduced, a corresponding improvement in mass limit of detection is realized. Therefore, the coupling of CES with microanalytical methods is likely the largest area of opportunity for future research efforts within this field of study.

\section{Conflicts of Interest}

The author declares that there is no conflict of interest regarding the publication of this paper.

\section{References}

[1] L. C. Brent, W. J. Thorn, M. Gupta et al., "Evaluation of the use of a commercially available cavity ringdown absorption spectrometer for measuring $\mathrm{NO}_{2}$ in flight, and observations over the Mid-Atlantic States, during DISCOVER-AQ," Journal of Atmospheric Chemistry, vol. 72, no. 3-4, pp. 503-521, 2015.

[2] C. Jiang, M. Sun, Z. Wang et al., "Portable real-time ringdown breath acetone analyzer: Toward potential diabetic screening and management," Sensors, vol. 16, no. 8, article 1199, 2016.

[3] B. A. Paldus, C. C. Harb, T. G. Spence et al., "Cavity ringdown spectroscopy using mid-infrared quantum-cascade lasers," Optics Letters, vol. 25, no. 9, pp. 666-668, 2000.
[4] J. E. Thompson, P. L. Hayes, J. L. Jimenez et al., "Aerosol optical properties at Pasadena, CA during CALNEX 2010," Atmospheric Environment, vol. 55, pp. 190-200, 2012.

[5] Y. Wei, L. Ma, T. Cao et al., "Light scattering and extinction measurements combined with laser-induced incandescence for the real-time determination of soot mass absorption cross section," Analytical Chemistry, vol. 85, no. 19, pp. 9181-9188, 2013.

[6] J. E. Thompson, B. W. Smith, and J. D. Winefordner, "Atmospheric aerosol measurements by cavity ringdown turbidimetry," Aerosol Science and Technology, vol. 37, no. 3, pp. 221-230, 2003.

[7] K. Dial, S. Hiemstra, and J. E. Thompson, "Simultaneous measurement of optical scattering and extinction on dispersed aerosol samples," Analytical Chemistry, vol. 82, no. 19, pp. 7885-7896, 2010.

[8] L. Ma and J. E. Thompson, "Optical properties of dispersed aerosols in the near UV (355 nm): measurement approach and initial data," Analytical Chemistry, vol. 84, no. 13, pp. 5611-5617, 2012.

[9] S. Zadoo and J. E. Thompson, "Rayleigh scattering measurements of several fluorocarbon gases," Journal of Environmental Monitoring, vol. 13, no. 11, pp. 3294-3297, 2011.

[10] H. Naus and W. Ubachs, "Experimental verification of Rayleigh scattering cross sections," Optics Letters, vol. 25, no. 5, pp. 347-349, 2000.

[11] S. Kassi and A. Campargue, "Cavity ring down spectroscopy with $5 \times 10^{-13} \mathrm{~cm}^{-1}$ sensitivity," The Journal of Chemical Physics, vol. 137, no. 23, Article ID 234201, 2012.

[12] M. Mazurenka, A. J. Orr-Ewing, R. Peverall, and G. A. D. Ritchie, "Cavity ring- down and cavity enhanced spectroscopy using diode lasers," Annual Reports on the Progress of Chemistry, Section C: Physical Chemistry, vol. 101, 100 pages, 2005.

[13] R. Centeno, S. Mandon, S. M. Cristescu, and F. J. M. Harren, "Sensitivity enhancement in off-axis integrated cavity output spectroscopy," Optics Express, vol. 22, no. 23, pp. 27985-27991, 2014.

[14] J. E. Thompson and H. Spangler, “Tungsten source integrated cavity output spectroscopy (W-ICOS) for the determination of ambient atmospheric extinction coefficient," Applied Optics, vol. 45, no. 11, pp. 2465-2473, 2006.

[15] A. O'Keefe, J. J. Scherer, and J. B. Paul, "CW integrated cavity output spectroscopy," Chemical Physics Letters, vol. 307, no. 56, pp. 343-349, 1999.

[16] G. D. Reid, M. D. Robertson, and A. Z. Tong, "Derivation of new equations for phase-shift cavity ring-down spectroscopy," Journal of Physical Chemistry A, vol. 115, no. 19, pp. 4902-4908, 2011.

[17] Y. Sadanaga, R. Takaji, A. Ishiyama, K. Nakajima, A. Matsuki, and $\mathrm{H}$. Bandow, "Thermal dissociation cavity attenuated phase shift spectroscopy for continuous measurement of total peroxy and organic nitrates in the clean atmosphere," The Review of Scientific Instruments, vol. 87, no. 7, article 074102, 2016.

[18] C. Dhiman, M. S. Khan, and M. N. Reddy, "Phase-shift cavity ring down spectroscopy set-up for $\mathrm{NO}_{2}$ sensing: design and fabrication," Defence Science Journal, vol. 65, no. 1, pp. 25-30, 2015.

[19] P. Massoli, P. L. Kebabian, T. B. Onasch, F. B. Hills, and A. Freedman, "Aerosol light extinction measurements by cavity attenuated phase shift (CAPS) spectroscopy: laboratory validation and field deployment of a compact aerosol particle extinction monitor," Aerosol Science and Technology, vol. 44, no. 6, pp. 428-435, 2010. 
[20] M. Islam, L. N. Seetohul, and Z. Ali, "Liquid phase broadband cavity enhanced spectroscopy measurements in a $2 \mathrm{~mm}$ cuvette," Applied Spectroscopy, vol. 61, no. 6, pp. 649-658, 2007.

[21] L. N. Seetohul, Z. Ali, and M. Islam, "Liquid-phase broadband cavity enhanced absorption spectroscopy (BBCEAS) studies in a $20 \mathrm{~cm}$ cell," Analyst, vol. 134, no. 9, pp. 1887-1895, 2009.

[22] S.-S. Kiwanuka, T. Laurila, and C. F. Kaminski, "Sensitive method for the kinetic measurement of trace species in liquids using cavity enhanced absorption spectroscopy with broad bandwidth supercontinuum radiation," Analytical Chemistry, vol. 82, no. 17, pp. 7498-7501, 2010.

[23] Z. Bajuszova, Z. Ali, S. Scott, L. N. Seetohul, and M. Islam, "Cavity-enhanced immunoassay measurements in microtiter plates using BBCEAS," Analytical Chemistry, vol. 88, no. 10, pp. 5264-5270, 2016.

[24] Y. Arai, T. Yamamoto, T. Minamikawa, T. Takamatsu, and T. Nagai, "Spectral fingerprinting of individual cells visualized by cavity reflection enhanced light absorption microscopy," PloS One, vol. 10, no. 5, e0125733 pages, 2015.

[25] T. Von Lerber and M. W. Sigrist, "Cavity-ring-down principle for fiber-optic resonators: experimental realization of bending loss and evanescent-field sensing," Applied Optics, vol. 41, no. 18, pp. 3567-3575, 2002.

[26] L. van der Sneppen, F. Ariese, C. Gooijer, and W. Ubachs, "Liquid-phase and evanescent-wave cavity ring-down spectroscopy in analytical chemistry," Annual Review of Analytical Chemistry, vol. 2, pp. 13-35, 2009.

[27] D. E. Vogler, M. G. Muller, and M. W. Sigrist, "Fiber-optic cavity sensing of hydrogen diffusion," Applied Optics, vol. 43, no. 3-5, pp. 527-535, 2003.

[28] D. E. Vogler, A. Lorencak, J. M. Rey, and M. W. Sigrist, "Bending loss measurement using a fiber cavity ringdown scheme," Optics and Lasers in Engineering, vol. 43, no. 3-5, pp. 527-535, 2005.

[29] N. L. P. Andrews, J. Litman, D. Stroh, J. A. Barnes, and H.-P. Loock, "Near-infrared absorption detection in picolitre liquid volumes using amplified fibre loop ring-down detection," Optical Fiber Technology, vol. 19, no. 6, pp. 822-827, 2013.

[30] C. Wang, "Fiber loop ringdown - a time-domain sensing technique for multi-function fiber optic sensor platforms: current status and design perspectives," Sensors, vol. 9, no. 10, pp. 7595-7621, 2009.

[31] K. Bescherer, J. A. Barnes, and H.-P. Loock, "Absorption measurements in liquid core waveguides using cavity ringdown spectroscopy," Analytical Chemistry, vol. 85, no. 9, pp. 4328-4334, 2013.

[32] Y. Zhao, L. Bai, B. Han, and Q. Wang, "Review on advances of sensors based on fiber loop ring-down spectroscopy," Instrumentation Science and Technology, vol. 41, no. 4, pp. 349-364, 2013.

[33] R. S. Brown, I. Kozin, Z. Tong, R. D. Oleschuk, and H.-P. Loock, "Fiber-loop ring-down spectroscopy," The Journal of Chemical Physics, vol. 117, no. 23, pp. 1044410447, 2002.

[34] C. M. Rushworth, D. James, J. W. L. Lee, and C. Vallance, "Top notch design for fiber-loop cavity ring-down spectroscopy," Analytical Chemistry, vol. 83, no. 22, pp. 8492-8500, 2011.

[35] H. Waechter, J. Litman, A. H. Cheung, J. A. Barnes, and H. P. Loock, "Chemical sensing using fiber cavity ring-down spectroscopy," Sensors, vol. 10, no. 3, pp. 1716-1742, 2010.
[36] M. Gupta, H. Jiao, and A. O'Keefe, "Cavity-enhanced spectroscopy in optical fibers," Optics Letters, vol. 27, no. 21, pp. 1878-1880, 2002.

[37] http://www.corning.com/media/worldwide/coc/documents/ Fiber/RC-\%20White\%20Papers/WP-General/WP1212_12-10 .pdf accessed September 9, 2016.

[38] G. Stewart, K. Atherton, H. B. Yu, and B. Culshaw, "An investigation of an optical fibre amplifier loop for intra-cavity and ring-down cavity loss measurements," Measurement Science and Technology, vol. 12, no. 7, pp. 843-849, 2001.

[39] G. Stewart, K. Atherton, H. B. Yu, and B. Culshaw, "Cavityenhanced spectroscopy in fiber cavities," Optics Letters, vol. 29, no. 5, pp. 442-444, 2004.

[40] J. E. Thompson and K. Myers, "Cavity ring-down lossmeter using a pulsed light emitting diode source and photon counting," Measurement Science and Technology, vol. 18, no. 1, pp. 147-154, 2007.

[41] P. B. Tarsa, P. Rabinowitz, and K. K. Lehmann, "Evanescent field absorption in a passive optical fiber resonator using continuous wave cavity ring-down spectroscopy," Chemical Physics Letters, vol. 383, no. 3, pp. 297-303, 2004.

[42] A. Nitkowski, L. Chen, and M. Lipson, "Cavity enhanced onchip absorption spectroscopy using microring resonators," Optics Express, vol. 16, no. 16, pp. 11930-11936, 2008.

[43] E. K. Sackmann, A. L. Fulton, and D. J. Beebe, "The present and future role of microfluidics in biomedical research," Nature, vol. 507, no. 7491, pp. 181-189, 2014.

[44] P. Li, Y. Gao, and D. Pappas, "Multiparameter cell affinity chromatography: separation and analysis in a single microfluidic channel," Analytical Chemistry, vol. 84, no. 19, pp. 8140-8148, 2012.

[45] P. Li, Y. Tian, and D. Pappas, "Comparison of inlet geometry in microfluidic cell affinity chromatography," Analytical Chemistry, vol. 83, no. 3, pp. 774-781, 2011.

[46] D. M. Birney and S. D. Starnes, "Parallel combinatorial esterification: a simple experiment for use in the second-semester organic chemistry laboratory," Journal of Chemical Education, vol. 76, no. 11, pp. 1560-1561, 1999.

[47] A. J. Alexander, "Flowing liquid-sheet jet for cavity ring-down absorption measurements," Analytical Chemistry, vol. 78, no. 15, pp. 5597-5600, 2006.

[48] S. E. Fiedler, A. Hese, and A. A. Ruth, "Incoherent broad-band cavity-enhanced absorption spectroscopy of liquids," The Review of Scientific Instruments, vol. 76, no. 2, Article ID 023107, 2005.

[49] Z. Qu, J. Engstrom, D. Wong, M. Islam, and C. F. Kaminski, "High sensitivity liquid phase measurements using broadband cavity enhanced absorption spectroscopy (BBCEAS) featuring a low cost webcam based prism spectrometer," Analyst, vol. 138, no. 21, pp. 6372-6379, 2013.

[50] K. L. Snyder and R. N. Zare, "Cavity ring-down spectroscopy as a detector for liquid chromatography," Analytical Chemistry, vol. 75, no. 13, pp. 3086-3091, 2003.

[51] S. Xu, G. Sha, and J. Xie, "Cavity ring-down spectroscopy in the liquid phase," The Review of Scientific Instruments, vol. 73, no. 2, pp. 255-258, 2002.

[52] B. Bahnev, L. van der Sneppen, A. E. Wiskerke, F. Ariese, C. Gooijer, and W. Ubachs, "Miniaturized cavity ring-down detection in a liquid flow cell," Analytical Chemistry, vol. 77, no. 4, pp. 1188-1191, 2005.

[53] T. McGarvey, A. Conjusteau, and H. Mabuchi, "Finesse and sensitivity gain in cavity-enhanced absorption spectroscopy 
of biomolecules in solution," Optics Express, vol. 14, no. 22, pp. 10,441-10,451, 2006.

[54] A. J. Hallock, E. S. F. Berman, and R. N. Zare, "Direct monitoring of absorption in solution by cavity ring-down spectroscopy," Analytical Chemistry, vol. 74, no. 7, pp. 1741$1743,2002$.

[55] A. J. Hallock, E. S. F. Berman, and R. N. Zare, "Use of broadband, continuous-wave diode lasers in cavity ring-down spectroscopy for liquid samples," Applied Spectroscopy, vol. 57, no. 5, pp. 571-573, 2003.

[56] H. Waechter, D. Munzke, A. Jang, and H. P. Loock, "Simultaneous and continuous multiple wavelength absorption spectroscopy on nanoliter volumes based on frequency-division multiplexing fiber-loop cavity ring-down spectroscopy," Analytical Chemistry, vol. 83, no. 7, pp. 2719-2725, 2011.

[57] H. Waechter, K. Bescherer, C. Durr, R. D. Oleschuk, and H. P. Loock, "405 nm absorption detection in nanoliter volumes," Analytical Chemistry, vol. 81, no. 21, pp. 9048-9054, 2009.

[58] D. James, B. Oag, C. M. Rushworth et al., "High-sensitivity online detection for microfluidics via cavity ringdown spectroscopy," RSC Advances, vol. 2, no. 12, pp. 5376-5384, 2012.

[59] R. D. Waterbury, W. S. Yao, and R. H. Bryne, "Long pathlength absorbance spectroscopy: trace analysis of $\mathrm{Fe}(\mathrm{II})$ using a $4.5 \mathrm{~m}$ liquid core waveguide," Analytica Chimica Acta, vol. 357, no. 1, pp. 99-102, 1997.

[60] S. K. Mishra and P. K. Dasgupta, "Capillary scale light emitting diode based multi-reflection absorbance detector," Analytica Chimica Acta, vol. 605, no. 2, pp. 166-174, 2007. 

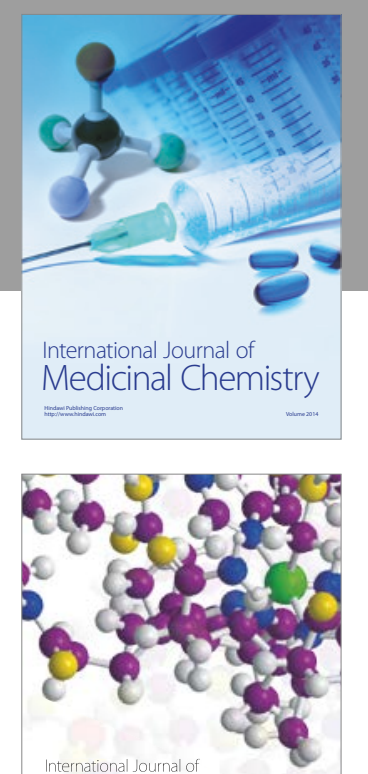

Carbohydrate Chemistry

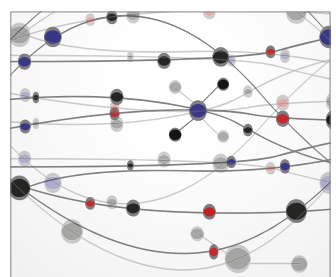

The Scientific World Journal

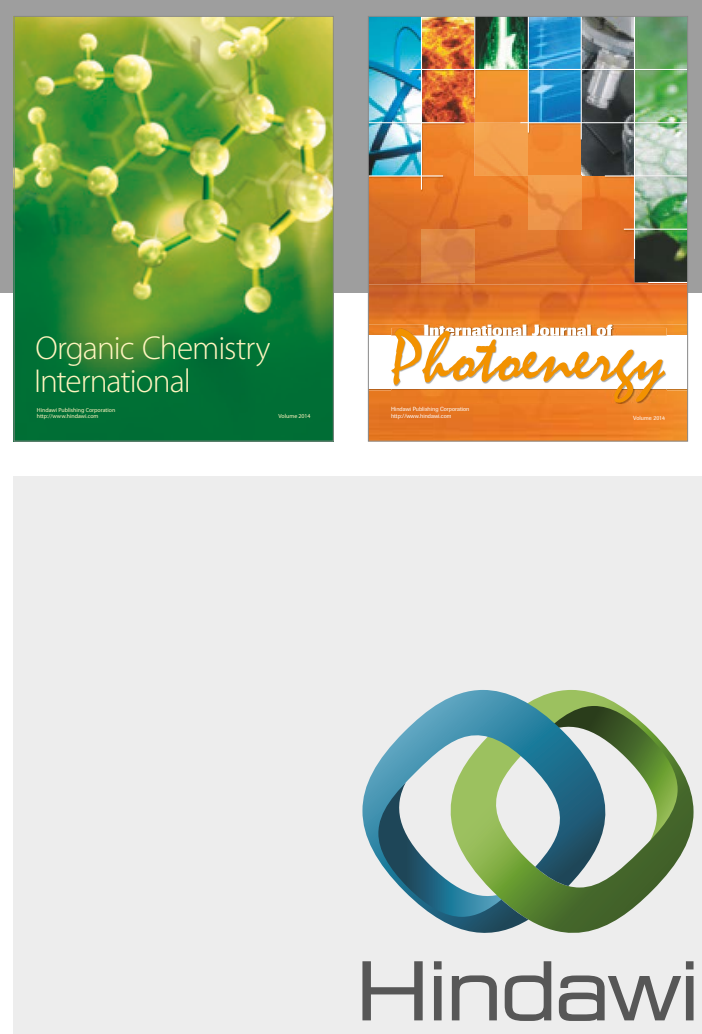

Submit your manuscripts at

https://www.hindawi.com

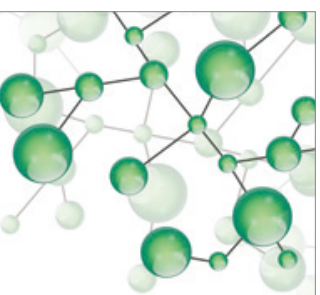

International Journal of

Inorganic Chemistry

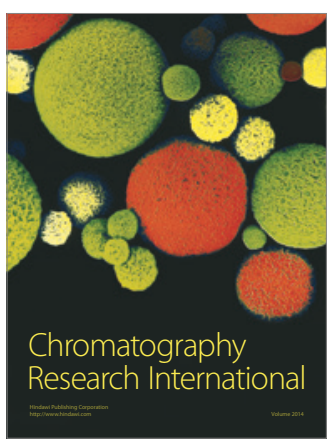



Applied Chemistry
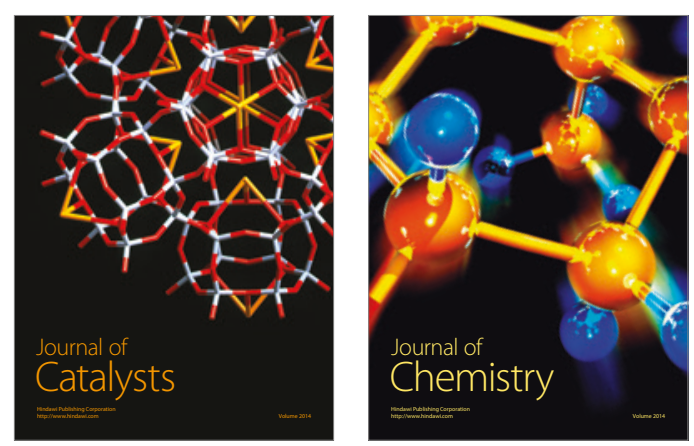
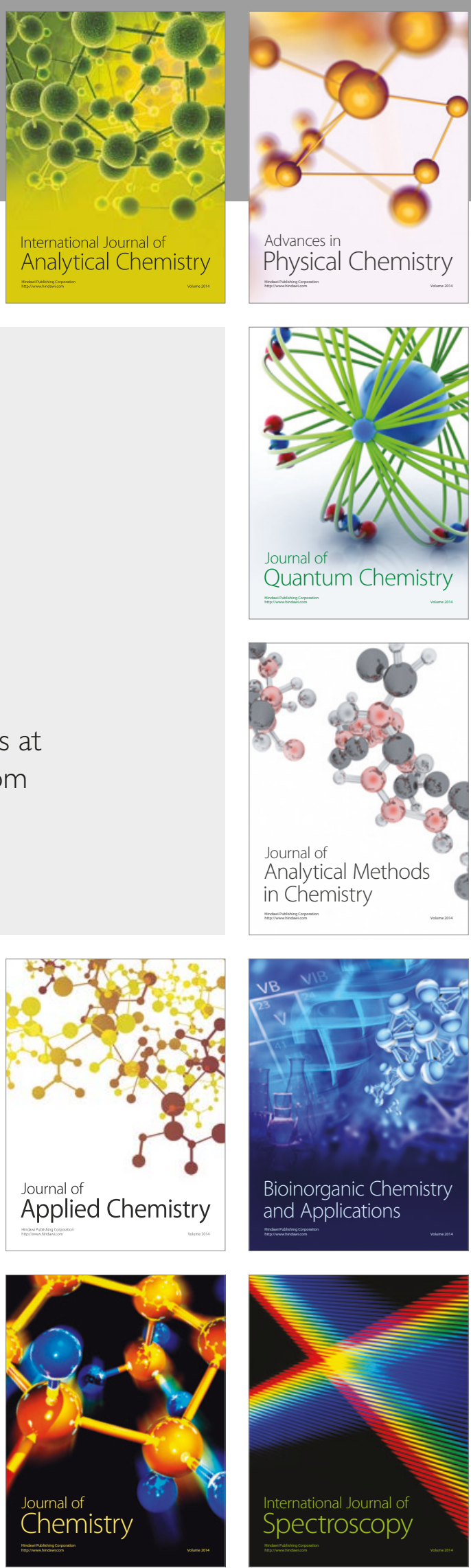